\title{
Outcome of in vitro fertilization in women with subclinical hypothyroidism
}

\author{
YunYing Cai ${ }^{1,2}$, LanPing Zhong ${ }^{3}$, Jie Guan ${ }^{3}$, RuiJin Guo ${ }^{2}$, Ben Niư ${ }^{2}$, YanPing Ma ${ }^{3}$ and Heng Su ${ }^{1,2^{*}}$
}

\begin{abstract}
Background: Previous studies examining associations between subclinical hypothyroidism (SCH) with in vitro fertilization (IVF) outcome indicate some benefits of levothyroxine (LT4) treatment. But IVF outcomes in treated SCH women whose serum Thyroid Stimulating Hormone (TSH) concentration did and did not exceed $2.5 \mathrm{mIU} / \mathrm{L}$ before the IVF cycle has not been studied thoroughly.

Methods: In this study, we performed a prospective cohort study with 270 treated subclinical hypothyroidism patients undergoing their first IVF retrieval cycle at a single cite.

Results: SCH in women receiving LT4 replacement with a basal TSH level between $0.2-2.5 \mathrm{mIU} / \mathrm{L}$ displayed a similar rate of clinical pregnancy $(47.4 \%$ vs $38.7 \%, P=.436)$, miscarriage $(7.4 \%$ vs $16.7 \%, P=.379)$ and live birth (43.9\% vs $32.3 \%, P=.288$ ) compared to women with a basal TSH level between $2.5-4.2 \mathrm{mIU} / \mathrm{L}$.

Conclusion: Strictly controlled TSH (less than $2.5 \mathrm{mIU} / \mathrm{L}$ ) before IVF may have no effect on the pregnancy rate in LT4 treated SCH women.

Keywords: Thyroid function, in vitro fertilization, Controlled ovarian stimulation, Subclinical hypothyroidism, Infertility
\end{abstract}

\section{Background}

Subclinical hypothyroidism $(\mathrm{SCH})$ is a common mild thyroid disorder in women of childbearing age and may contribute to increased risk of infertility and adverse obstetric outcome of pregnancy. Observational studies examined the effect of SCH on pregnancy related outcomes, and it has been associated with multiple adverse outcomes in many, but not all, studies [1]. A recent multicenter, randomized, placebo-controlled trial found that pregnant women with treated $\mathrm{SCH}$ whose median $\mathrm{TSH}$ was 4.2 $\mathrm{mIU} / \mathrm{l}$ are at the same frequencies for pregnancy loss, placental abruption, premature rupture of membranes, and neonatal death compared with untreated women [2]. Although unsound evidence exists,several professional organizations have maintained to recommend routine prenatal screening for and treatment of $\mathrm{SCH}$ during pregnancy $[3,4]$.

\footnotetext{
* Correspondence: su_hen@hotmail.com

${ }^{1}$ Faculty of Environmental Science and Engineering, Kunming University of Science and Technology, Kunming, Yunnan Province 650500, People's Republic of China

2Department of Endocrinology, The Affiliated Hospital of Kunming University of Science and Technology, The First People's Hospital of Yunnan, Kunming, Yunnan Province 650032, People's Republic of China

Full list of author information is available at the end of the article
}

The alteration of serum estrogen level during pregnancy has a profound physiological impact on thyroid function. Production of thyroid hormone increases by approximately $50 \%$ during pregnancy as a normal physiological response [5]. The functional reserve of thyroid is poor in hypothyroid women substituted with levothyroxine (L-T4) and they need to increase dose of medication to maintain normal thyroid function during pregnancy. Controlled ovarian stimulation $(\mathrm{COH})$ is a standard component of in vitro fertilization (IVF), which is able to induce a supraphysiological maternal serum estradiol level and may have a greater effect on thyroid function than natural conception $[6,7]$. Several investigations showed that IVF had an effect on serum TSH level in L-T4 treated hypothyroid women [8]. Moreover,the majority of evidence shows that SCH has a negative effect on outcome of IVF [9]. However, whether this negative influence may be attenuated with LT4 treatment, the benefits of strictly controlled TSH level before IVF are still unknown $[3,9]$. Our aim was to investigate the rate of pregnancies of IVF in $\mathrm{SCH}$ women substituted with L-T4 and evaluate whether TSH less than $2.5 \mathrm{mIU} / \mathrm{l}$ is the optimal cut-off point before IVF cycle. We performed a prospective cohort study with patients 
undergoing their first IVF retrieval cycle at a single cite from January 2015 to March 2016 (15 months).

\section{Methods \\ Patients}

Four thousand and seven hundred twenty subfertile women who underwent either classical IVF or IVFIntracytoplasmic Sperm Injection (ICSI) were enrolled between January 2015 and March 2016 at the first people's hospital of Yunnan Province PR China. The level of TSH was screened and the subjects with TSH level above $4.2 \mathrm{mIU} / \mathrm{L}$ were selected, serum levels of TSH, free triiodothyronine (FT3), free thyroxine (FT4) were measured. SCH is defined as an elevated TSH concentration (TSH 4.2-20 mIU/L) with normal serum levels of FT4. When $\mathrm{SCH}$ was confirmed, treatment with L-T4 was started and the target TSH level is within 0.2-4.2 $\mathrm{mIU} / \mathrm{l}$. After the approval of Institutional Review Board, we selected women aged 37 years or less who underwent their first fresh IVF retrieval cycle, using their own oocytes. Two hundred and seventy $\mathrm{SCH}$ women substituted with LT4 were considered for further study. They were eligible if serum TSH level was within $0.2-4.2 \mathrm{mIU} / \mathrm{L}$ one month proceeding the IVF cycle. Age-matched euthyroid women who underwent either classical IVF or ICSI were selected as control group in the same period. This analysis exclusively refers to the first treatment cycle.

During $\mathrm{COH}$, participants were monitored closely with transvaginal ultrasound and serum hormone levels. When a lead follicle R18 $\mathrm{mm}$ in size was identified, intramuscular hCG was administered with oocyte retrieval 36 hours later. Three to 5 days after retrieval, embryo transfer (ET) was performed, and participants returned 14 days later for measuring serum hCG levels. Positive pregnancy test was defined as positive serum hCG, and participants were followed to determine pregnancy outcome (10 months). Samples were processed and serum stored at $-20{ }^{\circ} \mathrm{C}$ for batched analysis. Exclusion criteria included those patients with ovarian hyperstimulation (E2 $>4000 \mathrm{pg} / \mathrm{ml}$, the number of follicles over 20), poor ovarian response (less than three follicles after ovarian stimulating drugs), poor compliance. Clinical endpoints were rates of clinical pregnancy (gestational sac on ultrasound), miscarriage (loss after sonographic presence of gestational sac), and live birth (delivery confirmed via Society for Assisted Reproductive Technology and direct patient report).

\section{Laboratory Analysis}

The levels of serum TSH, FT4, FT3 and thyroid peroxidase antibody (TPOAb) were measured with electrochemiluminescence (ECL) immunoassays (CobasElesys 601, Roche). The TSH assay had a reference range of $0.27-4.2 \mathrm{mIU} / \mathrm{L}$ with intra-assay coefficient of variation (CV) of $1.57-4.12 \%$. For FT4, the reference range was
$12-22 \mathrm{pmol} / \mathrm{L}$ with intra-assay $\mathrm{CV}$ of $2.24 \%-6.33 \%$. The FT3 reference range was $3.1-6.8 \mathrm{pmol} / \mathrm{L}$ with intra-assay $\mathrm{CV}$ of $2.42 \%-5.63 \%$. The TPOAb assay range was 5.0 $1,000 \mathrm{IU} / \mathrm{mL}$ with intra-assay $\mathrm{CV}$ of $6.7 \%$. Positive TPOAb status was $>35 \mathrm{IU} / \mathrm{mL}$. Positive pregnancy test was defined by serum $\beta$-hCG more than $25 \mathrm{mIU} / \mathrm{ml}$, typically on day $12-14$ after oocyte retrieval.

\section{Statistical analysis}

Quantitative values were expressed as mean \pm SD or as median and interquartile range, as appropriate. Wilcoxon Rank-Sum test (nonparametric analysis) was used for continuous data without a normal distribution; ChiSquare analysis was used for categorical data with large cell counts, the Fisher-Exact test analysis for categorical data with small cell counts, a $p$-value $<0.05$ was considered statistically significant.

\section{Results}

We found in a cohort of 4720 subfertile women undergoing IVF that $5.8 \%$ of them had $\mathrm{SCH}$. Two hundred and seventy $\mathrm{SCH}$ women were enrolled and substituted with L-T4. Ninety-four $\mathrm{SCH}$ women were excluded because their IVF cycle were cancelled, one hundred and seventy-six of 270 women (66.2\%) completed all pregnancy study visits and included in the final analysis. Of the 4407 women $(93.3 \%)$ with normal thyroid screen results, 200 were eligible and participated in the study.

Baseline characteristics of the levothyroxine treated $\mathrm{SCH}$ group and the euthyroid group were similar. The frequencies of adverse pregnancy outcomes did not differ significantly between the two study groups (Table 1). Eighty-two of 176 (46.6\%) L-T4 treated SCH women who underwent ET had positive pregnancy tests. Seventy-eight of 176 (44.31\%) became clinically pregnant. Eight pregnancies $(10.3 \%)$ resulted in miscarriages (before 12 weeks of gestation). Overall rates of clinical pregnancy $(44.31 \%$ vs $38.36 \%, P=.251)$ and miscarriage $(10.3 \%$ vs $10.7 \%, P=.39)$ were not significantly different between L-T4 treated $\mathrm{SCH}$ women and euthyroid women who underwent IVF in the same period.

One hundred and fourteen of $176 \mathrm{~L}-\mathrm{T} 4$ treated $\mathrm{SCH}$ women $(64.8 \%)$ had a TSH level within $0.2-2.5 \mathrm{mIU} / \mathrm{L}$ before the IVF cycle. Fifty-four (47.4\%) of them had positive pregnancy tests and all of them had clinical pregnancies. Fifty (43.9\%) had a live birth and four (7.4\%) pregnancy resulted in miscarriage. Of the remaining 62 participants with a TSH level within $2.5-4.2 \mathrm{mIU} / \mathrm{L}$ before the IVF cycle, $28(45.2 \%)$ of them had positive pregnancy tests but only $24(38.7 \%)$ became clinically pregnant, 20 (32.3\%) had a live birth, 4 (16.7\%) resulting in miscarriages. The rates of clinical pregnancy $(P=.436)$, miscarriages $(P=.379)$ and live birth $(P=.288)$ were similar between two groups. In addition, total number of oocytes 
Table 1 Baseline characteristics and IVF outcomes in Treated SCH and Euthyroid Patients

\begin{tabular}{llll}
\hline Number of patients & $\begin{array}{l}\text { Sub-hypo women } \\
n=176\end{array}$ & $\begin{array}{l}\text { Euthyroid women } \\
n=200\end{array}$ & $P$ \\
\hline Age (yrs) & $29.89 \pm 4.07$ & $30.45 \pm 4.21$ & 0.056 \\
BMI(kg/m2) & $22.76 \pm 3.54$ & $22.41 \pm 2.89$ & 0.099 \\
TSH range(mIU/L) & $0.32,4.21$ & $0.57,4.21$ & 0.420 \\
Indication to IVF & & & 0.439 \\
Tubal factor infertility & $50 \%$ & $54.3 \%$ & \\
Ovulatory dysfunction & $7.95 \%$ & $5.9 \%$ & 0.059 \\
Endometriosis & $2.2 \%$ & $1.4 \%$ & \\
Male & $15.9 \%$ & $16.2 \%$ & \\
Mixed & $21.6 \%$ & $20 \%$ & \\
Unexplained infertility & $6.5 \%$ & $6.7 \%$ & 0.251 \\
Clinical pregnancies & $44.31 \%$ & $38.36 \%$ & 0.39 \\
Miscarriages & $10.3 \%$ & $10.7 \%$ & \\
\hline
\end{tabular}

retrieved $(P=.752)$, good quality embryos $(P=.752)$ were similar between these two groups. The baseline characteristics and cycle outcome of women whose serum TSH did and did not exceed the threshold of $2.5 \mathrm{mIU} / \mathrm{L}$ before the IVF cycle are shown in Table 2 . No statistically significant differences were observed. An intragroup analysis according to thyroid autoimmunity detected also failed to document any significant result (data not shown).

Subgroup analyses were performed within the group of women with treated SCH. We compared women with $(n=102)$ and without $(n=74)$ thyroid autoimmunity. The median serum TSH before the IVF cycle was 1.81 (0.95-3.69) and $1.71(0.95-2.69) \mathrm{mIU} / \mathrm{L}$, respectively $(p=0.22)$. Baseline characteristics of the two study groups were similar with the exception of total amount of recombinant FSH, which was slightly higher in women without thyroid autoimmunity $(1950.0 \pm 1650.0$ vs. 2400.0 $\pm 1950.0 \mathrm{IU}, p=0.031$ ). The main findings of the IVF outcomes are summarized in Table 3. No statistically significant differences emerged.

\section{Discussion}

Using the definition of TSH level greater than the upper limit of normal range (4-5mIU/L) with normal FT4 levels, the incidence of $\mathrm{SCH}$ has been reported approximately $4-8 \%$ in reproductive age population $[10,11]$. Similarly, the incidence of SCH during pregnancy ranged from 2 to $2.5 \%$ [3]. Some investigators reported that $\mathrm{SCH}$ is more prevalent in infertile women $[12,13]$. In a retrospective study, an elevated serum TSH was found in $4 \%$ of 335 infertile Finnish women with unknown history of thyroid dysfunction [8]. The incidence was highest in those with ovulatory dysfunction (6\%) and unexplained infertility [5]. In this study, we enrolled
4720 subfertile women who underwent IVF for thyroid function examination. The incidence of $\mathrm{SCH}$ was $5.8 \%$, using an upper cutoff TSH level of $4.2 \mathrm{mIU} / \mathrm{l}$, which is consistent with previous investigations [12, 13].

Multiple studies have reported an association of $\mathrm{SCH}$ with an increased risk of adverse pregnancy outcomes including placental abruption, preterm birth, fetal death, and preterm premature rupture of membranes (PPROM) $[3,14]$, but not all. There is fair evidence that $\mathrm{SCH}$, defined as TSH outside of the normal reference range in pregnancy, is associated with miscarriage $[3,15]$. Untreated $\mathrm{SCH}$ affected the pregnancy outcomes in women requiring IVF, such as decreased pregnancy rate, implantation rate and delivery rate. Current data have demonstrated some benefits of L-T4 treatment in reducing these events [9]. In 2007, investigators performed a randomized trial on a population of 70 infertile patients with SCH who had undergone IVF. Patients were randomly assigned to either the L-T4 treatment group (50-100 mc g L-T4 starting at one month before the assisted reproduction technology procedure) or the placebo group. They found that compared with untreated group, the miscarriage rate was significantly lower ( $9 \%$ vs $13 \%$, respectively) and the clinical pregnancy rate and delivery rate were significantly higher (35\% and $10 \%$ vs $26 \%$ and $3 \%$, respectively) in L-T4 treatment group[16]. Another study randomized 64 women with $\mathrm{SCH}$ to the L-T4 treatment group (50 mcg L-T4 starting at day 1 of ovarian stimulation) or the control group [17]. They observed a significant increase in the number of grade I or II embryos $(P=.007)$ and in the implantation rate $(26.9 \%$ vs $14.9 \%, P=.044)$. The miscarriage rate was significantly lower in the L-T4 treatment group ( 0 vs $33.3 \% ; P=.021$ ) and as a result, the live-birth rate was significantly higher in the L-T4 treatment group (53.1\% vs $25 \%, P=.039$ ). In our study, $176 \mathrm{SCH}$ women were substituted with $\mathrm{L}-\mathrm{T} 4$ and their TSH levels were maintained within $0.2-4.2 \mathrm{mIU} / \mathrm{L}$ one month before the IVF cycle. We found that the rate of positive pregnancy test, clinical pregnancies and miscarriage were similar between treated $\mathrm{SCH}$ women and euthyroid women who underwent IVF, which is consistent with previous investigations. The investigation found that IVF outcome was not significantly hampered in women with adequately treated hypothyroidism [18].

There is powerful evidence that L-T4 treatment in women with $\mathrm{SCH}$ is associated with improvement in pregnancy and miscarriage rates [1], but, the benefit of thyroid hormone use on pregnancy loss was maily observed among women with basal TSH concentrations of 4.1-10.0 mIU/L, not those with concentrations of 2.5$4.0 \mathrm{mIU} / \mathrm{L}$. Furthermore, the benefits of strict TSH control remains to be determined [4, 19-21]. Few studies have examined if the risks of adverse obstetric outcomes 
Table 2 Baseline characteristics and IVF outcomes in women serum TSH concentration did and did not exceed $2.5 \mathrm{mIU} / \mathrm{L}$ before the IVF cycle

\begin{tabular}{|c|c|c|c|}
\hline \multirow[t]{2}{*}{ Characteristics } & \multirow{2}{*}{$\begin{array}{l}\mathrm{TSH} \geq 2.5 \mathrm{mIU} / \mathrm{L} \\
n=62\end{array}$} & \multirow{2}{*}{$\begin{array}{l}\mathrm{TSH}<2.5 \mathrm{mIU} / \mathrm{L} \\
n=114\end{array}$} & \multirow[t]{2}{*}{$P$} \\
\hline & & & \\
\hline Age (yrs) & $30.48 \pm 4.07$ & $29.56 \pm 4.37$ & 0.336 \\
\hline $\mathrm{BMI}(\mathrm{kg} / \mathrm{m} 2)$ & $23.60 \pm 4.05$ & $22.29 \pm 3.18$ & 0.099 \\
\hline Previous pregnancies & $48.4 \%$ & $54.4 \%$ & \\
\hline AFC & $12.00 \pm 5.00$ & $12.00 \pm 8.00$ & 0.972 \\
\hline $\mathrm{TSH}(\mathrm{mlU} / \mathrm{L})$ & $2.60,4.21$ & $0.32,1.88$ & 0.0 \\
\hline FT3(pmol/L) & $4.73 \pm 0.76$ & $5.10 \pm 0.99$ & 0.005 \\
\hline FT4(pmol/L) & $17.29 \pm 2.29$ & $20.10 \pm 3.99$ & 0.001 \\
\hline Indication to IVF & & & 0.439 \\
\hline Tubal factor infertility & $45.2 \%$ & $52.6 \%$ & \\
\hline Ovulatory dysfunction & $6.4 \%$ & $8.8 \%$ & \\
\hline Endometriosis & $3.2 \%$ & $0 \%$ & \\
\hline Male & $12.9 \%$ & $17.5 \%$ & \\
\hline Mixed & $25.8 \%$ & $19.3 \%$ & \\
\hline Unexplained infertility & $6.5 \%$ & $1.8 \%$ & \\
\hline \multicolumn{4}{|l|}{ Protocol of stimulation } \\
\hline Long protocol & $77.4 \%$ & $89.5 \%$ & \\
\hline Short protocol & $6.5 \%$ & $3.5 \%$ & \\
\hline GnRH antagonists & $3.2 \%$ & $0 \%$ & \\
\hline ART procedure & & & 0.8 \\
\hline IVF & $75.8 \%$ & $70.2 \%$ & \\
\hline$|\mathrm{CS}|$ & $24.2 \%$ & $29.8 \%$ & \\
\hline Duration of stimulation(Days) & $11.23 \pm 1.82$ & $11.98 \pm 2.09$ & 0.106 \\
\hline Total amount of recombinant FSH administration & $2025 \pm 825$ & $2475.00 \pm 1350.00$ & 0.128 \\
\hline Serum estradiol at HCG Administration(pg/ml) & $1983.50 \pm 2079$ & $2529.00 \pm 2005.00$ & 0.108 \\
\hline Total number of oocytes retrieved & $10.00 \pm 8.00$ & $11.00 \pm 8.00$ & 0.752 \\
\hline Total number of good quality embryos & $2.00 \pm 3.00$ & $4.00 \pm 6.00$ & 0.767 \\
\hline Clinical pregnancies & $38.7 \%$ & $47.4 \%$ & 0.436 \\
\hline Miscarriages & $16.7 \%$ & $7.4 \%$ & 0.379 \\
\hline Live birth rate & $32.3 \%$ & $43.9 \%$ & 0.288 \\
\hline
\end{tabular}

Data are reported as $\%$,mean \pm standard deviation or quartile,as appropriate

AFC antral follicle, BMI, body mass index, FSH follicle-stimulating hormone, GnRH gonadotropin-releasing hormone

are increased with a TSH level higher than $2.5 \mathrm{mIU} / \mathrm{L}$ but less than the upper range of normal at the time of conception in IVF. Although unsound evidence exists recent Clinical Practice Guideline by The Endocrine Society suggests TSH levels in subclinical women should be less than $2.5 \mathrm{mIU} / \mathrm{L}$ before pregnancy, particularly in women requiring IVF [4]. One study measured 1231 women pursuing assisted reproductive technologies (ART). In this study, investigators found no evidence of an elevated pregnancy loss rate in women with preconception elevated TSH (2.5-4.0 mIU/L) [22]. The observation is in agreement with a recent study by Reh et al. who found no difference in clinical pregnancy, delivery, or miscarriage in patients with mildly elevated basal TSH $(>2.5$ and $<4.0$ $\mathrm{mIU} / \mathrm{L})$ compared to patients with a normal TSH $(>0.4$ to $<2.5 \mathrm{mIU} / \mathrm{L}$ ) [20]. Other studies suggest that there is a trend toward increasing risk of miscarriage with increasing TSH level, although this trend did not reach statistical significance [21]. These controversal results raise questions about the current guideline recommended threshold of $2.5 \mathrm{mIU} / \mathrm{L}$ for treating SCH when thyroid antibody was positive. In our study, no differences in the rates of positive pregnancy test, clinical pregnancies, miscarriage or live birth were observed in treated $\mathrm{SCH}$ women with different TSH level. There was no significant difference between the two groups with the number of oocytes 
Table 3 Baseline characteristics and IVF outcomes in women with treated subclinical hypothyroidism with or without thyroid antibodies

\begin{tabular}{llll}
\hline Characteristics & AB POS & AB NEG \\
& $n=102$ & $n=74$ \\
\hline Protocol of stimulation & & \\
Long protocol & $88.3 \%$ & $97.3 \%$ \\
Short protocol & $3.9 \%$ & $2.7 \%$ & $0 \%$ \\
GnRH antagonists & $7.8 \%$ & & \\
ART procedure & & $81.1 \%$ & $18.9 \%$ \\
IVF & $70.6 \%$ & $12.0 \pm 10.25$ \\
ICSI & $29.4 \%$ & $2400.0 \pm 1950.0$ \\
Duration of stimulation(Days) & $11.0 \pm 10.0$ & $2269.04 \pm 1239.37$ \\
Total amount of recombinant FSH administration & $1950.0 \pm 1650.0$ & $11.00 \pm 7.25$ \\
Serum estradiol at HCG Administration(pg/ml) & $2566.36 \pm 1162.43$ & $3.00 \pm 1.00$ \\
Total number of oocytes retrieved & $10.00 \pm 6.00$ & 0.241 \\
Total number of good quality embryos & $2.00 \pm 1.00$ & $40.6 \%$ & 0.031 \\
Clinical pregnancies & $47.1 \%$ & $18.2 \%$ & 0.34 \\
Miscarriages & $9.0 \%$ & $34.4 \%$ & 0.922 \\
Live birth rate & $43.1 \%$ & 0.965 \\
\hline
\end{tabular}

retrieved and in the implantation rate. In addition, we also use TSH values greater than $2.5 \mathrm{mIU} / \mathrm{L}$ on HCG admistration day as a cut-off point. There were no differences in the rates of positive pregnancy test, clinical pregnancies, miscarriage or live birth in different groups (data not shown).

However, due to the small sample size, the statistical power of this study was limited. In addition, since we didn't adjust the levothyroxine dose during $\mathrm{COH}$, we cannot determine whether there is an association between TSH alteration during ART and IVF outcome. We didn't measure urinary iodine in this study that was also a flaw. Finally, according to ATA guideline, we arbitrarily chose $2.5 \mathrm{mIU} / \mathrm{L}$ as a strictly controlled TSH threshold. It would have been more appropriate to refer to a local threshold since reference range may vary among different ethnics and is not always correct to use the same threshold for all ethnics.

\section{Conclusion}

In conclusion, our results suggest that L-T4 treatment in women with $\mathrm{SCH}$ requiring IVF had similar rates of pregnancy and adverse pregnancy outcomes compared to euthyroid women. Strictly controlled TSH (less than $2.5 \mathrm{mIU} / \mathrm{L}$ ) before IVF may have no effect on the pregnancy rate and maternal adverse pregnancy results. Whether the risk of other adverse outcomes could be attenuated calls for further studies to evaluate the effectiveness of thyroid hormone treatment in this population.

\section{Abbreviations}

ART: Assisted Reproductive Technology; $\mathrm{COH}$ : Controlled ovarian stimulation; ET: Embryo Transfer; FT3: Free Triiodothyronine; FT4: Free Thyroxine;

ICSI: Intracytoplasmic Sperm Injection; IVF: in vitro fertilization; L-T4: Levothyroxine; $\mathrm{SCH}$ : Subclinical hypothyroidism

\section{Acknowledgement}

The authors thank the staff of Reproductive Medicine Center and Endocrinology Laboratory of The First People's Hospital of Yunnan Province P.R. China for sample collection.

\section{Funding}

The present study was supported by the Scientific Research Fund Project of Department of Education in Yunnan Province (NO.2016ZDX015) and highlevel health professionals training projects of Yunnan Province (L-201624).

\section{Availability of data and materials}

The datasets used and/or analysed during the current study available from the corresponding author on reasonable request.

\section{Authors' contributions}

YC and HS wrote the manuscript. YC, LZ and HS designed the experiments; $Y C, L Z, R G$ and BN performed the experiments; BN, LZ,YP and JG participated in the analysis and discussion of the results. All authors read and approved the final version of the manuscript.

\section{Competing interests}

The authors declare that they have no competing interests.

\section{Consent for publication}

Not applicable.

\section{Ethics approval and consent to participate}

All experiments were performed in strict accordance with the Ethics Committee at The First People's Hospital of Yunnan P.R. China. Informed consent was obtained from all subjects. The Institutional Committee of the First People's Hospital of Yunnan P.R. China approved the experimental protocols (registration number: 2015092003).

\section{Publisher's Note}

Springer Nature remains neutral with regard to jurisdictional claims in published maps and institutional affiliations.

\section{Author details}

${ }^{1}$ Faculty of Environmental Science and Engineering, Kunming University of Science and Technology, Kunming, Yunnan Province 650500, People's Republic of China. ${ }^{2}$ Department of Endocrinology, The Affiliated Hospital of 
Kunming University of Science and Technology, The First People's Hospital of Yunnan, Kunming, Yunnan Province 650032, People's Republic of China. ${ }^{3}$ Reproductive Medicine Center, The Affiliated Hospital of Kunming University of Science and Technology, The First People's Hospital of Yunnan, Kunming, Yunnan 650032, People's Republic of China.

Received: 29 March 2017 Accepted: 14 May 2017

Published online: 25 May 2017

\section{References}

1. Maraka S, Mwangi R, McCoy RG, Yao X, Sangaralingham LR, Singh Ospina NM, O'Keeffe DT, De Ycaza AE, Rodriguez-Gutierrez R, Coddington CC, Stan MN, Brito JP, Montori VM. Thyroid hormone treatment among pregnant women with subclinical hypothyroidism: US national assessment. BMJ. 2017; 356:16865. PMID: 28122781.

2. Casey BM, Thom EA, Peaceman AM, Varner MW, Sorokin Y, Hirtz DG, Reddy UM, Wapner RJ, Thorp Jr JM, Saade G, Tita AT, Rouse DJ, Sibai B, lams JD, Mercer BM, Tolosa J, Caritis SN, VanDorsten JP. Treatment of subclinical hypothyroidism or hypothyroxinemia in pregnancy. N Engl J Med. 2017; 376(9):815-25. PMID: 28249134

3. Practice Committee of the American Society for Reproductive Medicine. Subclinical hypothyroidism in the infertile female population: a guideline[J]. Fertil Steril. 2015;104(3):545-53. PMID: 26239023.

4. Alexander EK, Pearce EN, Brent GA, Brown RS, Chen H, Dosiou C, Grobman WA, Laurberg P, Lazarus JH, Mandel SJ, Peeters RP, Sullivan S. 2017 guidelines of the American thyroid association for the diagnosis and management of thyroid disease during pregnancy and the postpartum. Thyroid. 2017;27(3):315-89. PMID: 28056690.

5. De Groot L, Abalovich 9, Alexander EK, et al. Management of thyroid dysfunction during pregnancy and postpartum: an Endocrine Society clinical practice guideline. J Clin Endocrinol Metab. 2012;97(8):2543-65. PMID: 22869843.

6. Meng Y, Lv PP, Ding GL, et al. High maternal serum estradiol levels induce dyslipidemia in human newborns via a hepatic HMGCR estrogen response element[J]. Sci Rep. 2015;5:10086. Published online 2015 May 11. PMCID: PMC4426719.

7. Reinblatt S, Herrero B, Correa JA, et al. Thyroid stimulating hormone levels rise after assisted reproductive technology[J]. J Assist Reprod Genet. 2013; 30(10):1347-52. PMCID: PMC3824851.

8. Busnelli A, Somigliana E, Benaglia $L$, et al. Thyroid axis dysregulation during in vitro fertilization in hypothyroid-treated patients. Thyroid. 2014;24(11): 1650-5. PMID: 25089619

9. Velkeniers B, Van Meerhaeghe A, Poppe K, et al. Levothyroxine treatment and pregnancy outcome in women with subclinical hypothyroidism undergoing assisted reproduction technologies: systematic review and meta-analysis of RCTs[J]. Hum Reprod Update. 2013;19(3):251-8. PMID: 23327883

10. Hollowell JG, Staehling NW, Flanders WD, et al. Serum TSH, T(4), and thyroid antibodies in the United States population (1988 to 1994): National Health and Nutrition Examination Survey (NHANES III). J Clin Endocrinol Metab. 2002;87(2):489-99. PMID: 11836274.

11. Canaris GJ, Manowitz NR, Mayor G, et al. The Colorado thyroid disease prevalence study. Arch Intern Med. 2000;160(4):526-34. PMID: 10695693.

12. Arojoki $M$, Jokimaa $V$, Juuti $A$, et al. Hypothyroidism among infertile women in Finland[J]. Gynecol Endocrinol. 2000;14(2):127-31. PMID: 10836200.

13. Lincoln SR, Ke RW, Kutteh WH. Screening for hypothyroidism in infertile women. J Reprod Med. 1999;44(5):455-7. PMID: 10360260.

14. Casey BM, Dashe JS, Wells CE, et al. Subclinical hypothyroidism and pregnancy outcomes. Obstet Gynecol. 2005;105(2):239-45. PMID: 15684146.

15. Maraka S, Ospina NM, O'Keeffe DT, et al. Subclinical Hypothyroidism in Pregnancy: A Systematic Review and Meta-Analysis. Thyroid. 2016;26(4): 580-90. PMID: 26837268.

16. Abdel Rahman AH, Aly Abbassy H, Abbassy AA. Improved in vitro fertilization outcomes after treatment of subclinical hypothyroidism in infertile women[J]. A Endocr Pract. 2010;16(5):792-7. PMID: 20350920.

17. Kim CH, Ahn JW, Kang SP, et al. Effect of levothyroxine treatment on in vitro fertilization and pregnancy outcome in infertile women with subclinical hypothyroidism undergoing in vitro fertilization/intracytoplasmic sperm injection[J]. Fertil Steril. 2011;95(5):1650-4. PMID: 21193190.
18. Busnelli A, Somigliana E, Benaglia L, Leonardi M, Ragni G, Fedele L. In vitro fertilization outcomes in treated hypothyroidism. Thyroid. 2013; 23(10):1319-25. PMID: 23544891.

19. Aghahosseini $M$, Asgharifard $H$, Aleyasin $A$, et al. Effects of Thyroid Stimulating Hormone (TSH) level on clinical pregnancy rate via In Vitro Fertilization (IVF) procedure[J]. Med J Islam Repub Iran. 2014;28:46. eCollection 2014. PMID: 25405112.

20. Reh A, Grifo J, Danoff A. What is a normal thyroid-stimulating hormone (TSH) level? Effects of stricter TSH thresholds on pregnancy outcomes after in vitro fertilization. Fertil Steril. 2010;94(7):2920-2. doi:10.1016/j.fertnstert. 2010.06.041. Epub 2010 Jul 23. PMID: 20655528.

21. Zeng $X$, Wang $L$, Shu $X$, et al. Influence of basic thyroid-stimulating hormone levels on outcomes of IVF/ICSI in Qinghai[J]. Zhonghua Fu Chan KeZa Zhi. 2014;49(10):763-77. PMID: 25537249.

22. Michalakis KG, Mesen TB, Brayboy LM, et al. Subclinical elevations of thyroid-stimulating hormone and assisted reproductive technology outcomes. Fertil Steril. 2011;95(8):2634-7. doi:10.1016/j.fertnstert.2011.02. 056. PMID: 21457968, Epub 2011 Mar 31.

\section{Submit your next manuscript to BioMed Central and we will help you at every step:}

- We accept pre-submission inquiries

- Our selector tool helps you to find the most relevant journal

- We provide round the clock customer support

- Convenient online submission

- Thorough peer review

- Inclusion in PubMed and all major indexing services

- Maximum visibility for your research

Submit your manuscript at www.biomedcentral.com/submit
) BioMed Central 